\title{
52. A Monoclonal Antibody against a Synthetic Polypeptide Fragment of Dystrophin (Amino Acid Sequence from Position 215 to 264)
}

\author{
By Teruo Shimizu,*),***) Kiichiro Matsumura,*),****) \\ Kazuki Hashimoto,*) Toru Mannen,*) Tsuneo Ishiguro,**),***) \\ Chikahiko EgUCHI,**).***) Ikuya NonAKA,***) Mikiharu YoshIdA,***) \\ and Eijiro OzAWA***)
}

(Communicated by Setsuro Ebashi, M. J. A., Sept. 12, 1988)

Recently, Koenig et al. have clarified the complete coding sequence of the Duchenne muscular dystrophy (DMD) gene transcript and deduced whole amino acid sequence of the protein named dystrophin. ${ }^{1)-3)}$ They calculated the molecular weight to be $427 \mathrm{kd}$. Three kinds of antisera against the protein fragment have shown that the immunoreactivity is associated with muscle sarcolemma, ${ }^{4)-7}$ ) especially its cytoplasmic face ${ }^{8)}$ in normal muscle, and reduced or absent in muscle fibers from DMD patients.

Here we established a monoclonal antibody against a synthetic peptide fragment from position 851 to 1000 on the human dystrophin cDNA map. The new probe demonstrated a very high molecular weight protein $(-400 \mathrm{kd})$ by Western blot analysis of human skeletal muscles. The antibody disclosed a chronological expression of the antigen at the surface membrane during human myogenesis and a variety of defective expression of the antigen in DMD muscles.

Materials and methods. The peptide fragment was synthetized by the solid phase method on Applied Biosystems model 430A from position 215 to 264 on the human dystrophin amino acid map deduced from cDNA nucleotide sequence, ${ }^{3}$ ) and purified with preparative reversed phase HPLC. The peptide corresponds to the initial part of the first repeating sequence of dystrophin. ${ }^{3}$ ) It consists of 50 amino acids, having a molecular weight of 5,775.

Balb/c mouse was immunized by a subcutaneous injection of $0.1 \mathrm{mg}$ of the peptide mixed with Freund's complete adjuvant and two boosters of $0.1 \mathrm{mg}$ of the peptide mixed in incomplete adjuvant with three-week interval. On the third day after the final booster, spleen cells were collected and fused with P3NP myeloma cell line under P4000 polyethylene glycol. Fifty-seven clones turned out to secrete an immunoglobulin $(\mathrm{s})$. Six clones secreted antibody which stained the surface membrane of human quadriceps femoris muscle. One of the six clones, $\mathrm{A} 1 \mathrm{C}$, was recloned and the monoclonal antibody (McAb) was characterized by Western blot analysis and indirect immunofluorescence microscopy.

For Western blot analysis, about $50 \mathrm{mg}$ of muscle was minced finely by

*) Department of Neurology, Institute of Brain Research, Faculty of Medicine, University of Tokyo, 7-3-1 Hongo, Bunkyo-ku, Tokyo, 113.

**) Central Research Laboratories, Ajinomoto Co., Inc., Kawasaki-ku, Kawasaki, 210 .

***) National Institute of Neuroscience, NCNP, Kodaira, Tokyo, 187.

****) Department of Neurology, Shimoshizu National Hospital, Yotsukaido, Chiba, 284. 


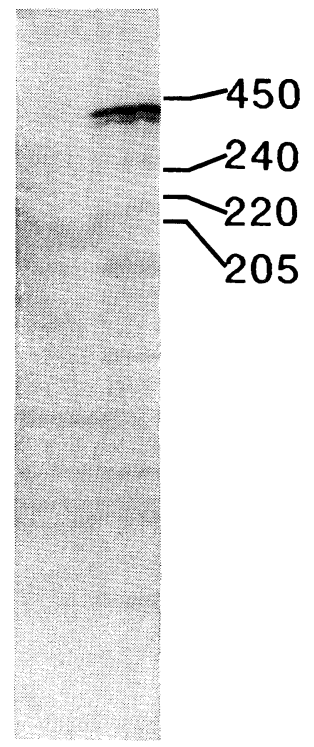

A B

Fig. 1. Western blot analysis of quadriceps femoris muscles from a DMD patient (lane A) and normal control (lane B) with $\mathrm{McAb}$ A1C. Molecular weight standards (laminin A: $450 \mathrm{kd}, \alpha$-spectrin: $240 \mathrm{kd}, \beta$-spectrin: $220 \mathrm{kd}$ and myosin heavy chain: $205 \mathrm{kd}$ ) are shown in the right side.

scissors in four volumes of the solution containing $70 \mathrm{mM}$ Tris- $\mathrm{HCl}(\mathrm{pH} \mathrm{6.7)}$, $10 \%$ sodium dodecylsulfate, $10 \%$ ethylenediaminetetraacetic acid, $5 \% \quad 2-$ mercaptoethanol and $10 \%$ glycerol. It was boiled for three minutes, left at rcom temperature overnight and clarified by centrifugation at $16,000 \times \mathrm{g}$ for five minutes. Five $\mu$ l of the supernatant was provided for SDS-PAGE. The methods of Doucet and Trifaro ${ }^{9)}$ and Kyhse-Anderson ${ }^{10)}$ were used for electrophoresis and electroblot, respectively. The proteins blotted on the nitrocellulose sheet were allowed to react with $\mathrm{McAb} \mathrm{A} 1 \mathrm{C}$ and the reaction was visualized with $\mathrm{ABC}$ kit (Vectastain, Vector Laboratories, Inc.).

For immunofluorescence microscopy, $8 \mu \mathrm{m}$ frozen sections of various muscle specimens were sequentially incubated with the antibody and FITC-labeled second antibody against mouse IgG. They were biopsied quadriceps femoris muscles from 19 patients with DMD, 8 patients with related neuromuscular diseases. The sections were observed under Zeiss epifluorescence microscope. For a study of chronological expression of dystrophin, biceps brachii muscles from legally-aborted fetuses were used.

Results. Western blot analysis showed that McAb A1C reacted with a protein from normal human muscle extract migrated between laminin $\mathrm{A}$ and $\alpha$-spectrin (Fig. 1B). A minor band preceding the protein was observed and it might be a proteolytic fragment. The molecular weight of the protein was approximately $400 \mathrm{kd}$ since the electrophoretic mobility was slightly larger than that of laminin A with the molecular weight of $450 \mathrm{kd} .{ }^{11)}$ The antibody also bound to a high molecular weight protein of rabbit back muscle, which corresponds to the human $400 \mathrm{kd}$ protein (data not shown). The protein was not detected in muscle extract from a DMD patient (Fig. 1A). In addition, the $\mathrm{McAb}$ crossreacted with human $\alpha$-spectrin (data not shown; Refs. 3), 12)), whereas no immunoreactivity was observed to other cytoskeletal proteins such as $\alpha$-actinin. ${ }^{3), 13)}$

Intracellular distribution of the antigen in muscle fibers was characterized 


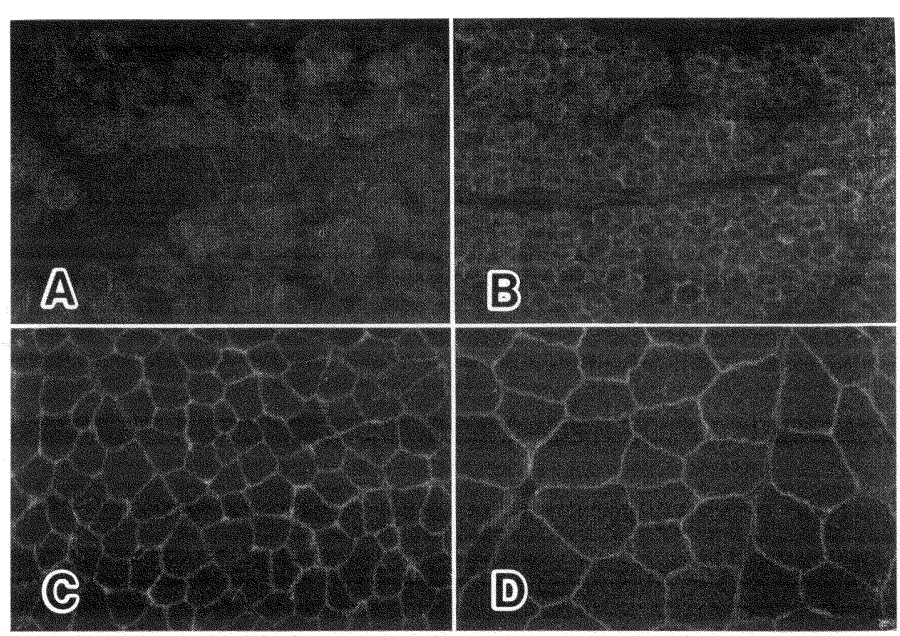

Fig. 2. Indirect immunofluorescent microscopy with biceps brachii muscles of 17-week (A) and 35-week (B) fetuses, 8-month infant (C) and adult control (D). Type 1, 2A, 2B and 2C fibers were detected in muscles shown in (C) and (D). $\times 180$.

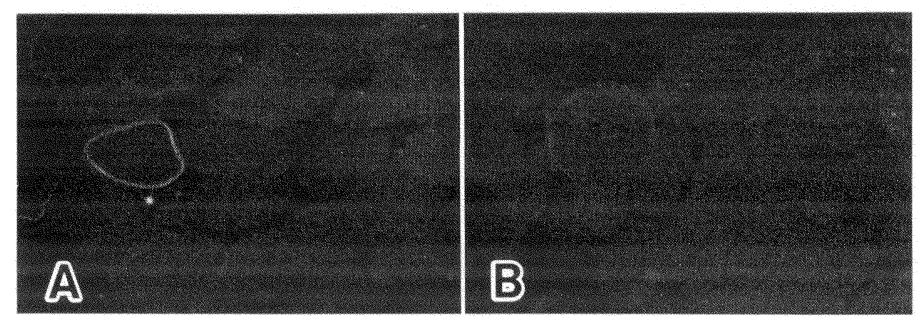

Fig. 3. Indirect immunofluorescent microscopy of quadriceps femoris muscles from DMD patients. Out of 19 patients examined, 16 patients showed a few immunofluorescent myofibers among many non-fluorescent fibers (A) and the other 3 patients demonstrated no immunoreactivity in examined areas (B). $\times 180$.

by indirect immunofluorescence microscopy (Fig. 2). The McAb stained continuously the whole surface membrane of myofibers in cross section from human quadriceps femoris (Fig. 2D) and rabbit hind limb muscles (data not shown). The longitudinal section showed no periodic staining corresponding to the repeat of sarcomere, but only continuous fluorescence throughout the whole length of the sarcolemma (data not shown). Biopsied muscles of 8 related neuromuscular diseases, including 2 cases of polymyositis, each one case of facioscapulohumeral muscular dystrophy, limb-girdle dystrophy, rimmed vacuole myopathy, myotonic dystrophy, amyotrophic lateral sclerosis and floppy infant showed a clear and definite staining at the sarcolemma. No immunoreactive material was detected in peripheral nerves. Biceps brachii muscles from 17- and 35-week-old fetuses and 8-month-old floppy boy were also analyzed for developmental expression of dystrophin. Some myofibers were partly stained in 17-week-old fetus (Fig. 2A), while in 35-week-old fetus most of myofibers were stained uniformly at sarcolemma (Fig. 2B). In 8-month-old boy every muscle fiber, including type 1, 
2A, 2B and 2C, was immunostained similarly to adult muscle (Fig. 2C).

In DMD, the immunoreactivity was hardly detected. Out of 19 DMD patients examined, myofibers from 3 patients were barely stained (Fig. 3B). A few myofibers from the other 16 patients were clearly stained, although a majority of fibers were not stained (Fig. 3A). The results demonstrate specificity and variety of defective expression of dystrophin in DMD.

Discussion. McAb A1C was obtained using a synthetic polypeptide fragment from amino acid position 215 to 264 predicted by $\mathrm{cDNA}$ sequence of human dystrophin. It reacted with a protein whose molecular weight was of approximately $400 \mathrm{kd}$ in immunoblotting analysis and immunocytochemically demonstrated the immunoreactive material at sarcolemma. In addition, the protein was hardly detected in myofibers from DMD patients. On the basis of the current knowledge, we conclude that McAb A1C is directed to dystrophin.

In human myogenesis, dystrophin was partly expressed at sarcolemma during a certain developing stage and fully expressed in well-differentiated muscle fibers. This is compatible with the developmental expression process of dystrophin in rat muscle (Hagiwara et al., in preparation). DMD muscles contain immature or regenerative type $2 \mathrm{C}$ fibers. The lack of immunoreactivity is not due to immaturity of fibers, since DMD muscles also contain many mature fibers. Myofibers from other related neuromuscular patients clearly reacted with $\mathrm{McAb}$ A1C. Therefore, the defective expression of dystrophin is specific in DMD.

Taking all these results into consideration, the nature of $\mathrm{McAb} \mathrm{A} 1 \mathrm{C}$ is close to that of the antiserum raised by Zubrzycka-Gaarn et al.5)

Acknowledgements. Thanks are due to Dr. H. Sugita for kind discussions and to Mmes. M. Kanno, S. Takeyama, T. Ogawa and E. Baba for their skillful technical assistance.

This work is supported partly by Grant-in-Aid for Scientific Research on Priority Areas, Ministry of Education, Science and Culture, and Grant (62-01 and 02) from the National Center of Neurology and Psychiatry (NCNP) of the Ministry of Health and Welfare, Japan.

\section{References}

1) Hoffman, E. P., Brown, R. H., and Kunkel, L. M.: Cell, 51, 919-928 (1987).

2) Hoffman, E. P. et al.: New England J. Med., 318, 1363-1368 (1988).

3) Koenig, M., Monaco, A. P., and Kunkel, L. M.: Cell, 53, 219-228 (1988).

4) Sugita, H. et al.: Proc. Japan Acad., 64B, 37-39 (1988).

5) Zubrzycka-Gaarn, E. et al.: Nature, 333, 466-469 (1988).

6) Arahata, K. et al.: ibid., 333, 861-863 (1988).

7) Bonilla, E. et al.: Cell, 54, 447-452 (1988).

8) Watkins, S. C. et al.: Nature, 333, 863-866 (1988).

9) Doucet, J. P., and Trifaro, J. M.: Anal. Biochem., 168, 265-271 (1988).

10) Kyhse-Anderson, J.: J. Biochem. Biophys. Meth., 10, 203-209 (1984).

11) Cooper, A. R. et al.: Eur. J. Biochem., 119, 189-197 (1981).

12) Davison, M. D., and Critchley, D. R.: Cell, 52, 159-160 (1988).

13) Hammonds, R. G. Jr.: ibid., 51, 1 (1987). 